\title{
Examining the Performance of a Constraint-Based Database Cache
}

\author{
Andreas Bühmann Joachim Klein \\ Databases and Information Systems, Department of Computer Science \\ University of Kaiserslautern, P. O. Box 3049, 67653 Kaiserslautern, Germany \\ buehmann@informatik.uni-kl.de_jklein@informatik.uni-kl.de
}

\begin{abstract}
Constraint-based database caching aims at correctly answering $S Q L$ query predicates from a local cache database by exploiting constraints that have previously been used in selecting sets of records to be cached from a remote database.

In this paper, we take our first steps in looking at performance aspects of our prototype Adaptive Constraint-based Cache (ACCache), which is realized in a middleware manner on top of regular databases. Within our measurement setup, the initial focus is on two central ACCache functions: query processing and cache loading. To demonstrate their time behavior and interaction, we have chosen a scenario based on the $T P C-W$ specification. We conclude with a discussion of our first measurement results.
\end{abstract}

\section{Motivation}

Applications that interact with real-world users typically strive for good (or at least acceptable) response times. This is a particular challenge if the application routinely relies on the services of a central backend database (DB) system that is located far from the application, e.g., in a Web scenario where application servers have been spread around the world at the "edge" of the Web to reduce their (network) distance to the users. In this scenario with usually a large number of users, relieving the backend system of some of its load becomes equally important.

Caching is a means to approach these two aims: By intercepting requests to a remote system component and constructing responses locally (from earlier responses or prefetched data), communication costs to and processing costs on the remote component can be saved. Caching can be performed on various levels within an information-system infrastructure: For example, generated Web pages (or fragments thereof) can be cached, persistent objects within an application server, or pages of a database in a DB buffer.

Database caching is located at the level of logical data structures (such as tables and records in a relational DB) and higher query languages (such as SQL). The goal is to have a cache in the path from the application to the backend DB that is as transparent as possible and that is able to process SQL queries locally based on locally stored parts of the backend DB. The constraintbased approach to database caching maintains a selection of cache tables, each containing a subset of records of the corresponding backend table. Cache constraints restrict what constitutes a valid state of the cache such that deciding what is in the cache and which predicates can be answered becomes easy.

\section{Constraint-based Database Caching}

In the general database-caching scenario, there are a backend (BE) database, which holds all data, and one or more cache databases, which contain varying subsets of that data. Ideally, the cache databases would contain data needed often in the nearer future.

With our model of constraint-based DB caching, cache groups are used to describe what data is to be kept in the cache and what constraints the cache contents have to fulfill at any time. These constraints can later be utilized to reason about whether a query can be (partly) answered from the cache.

For selected backend tables $T_{\mathrm{B}}$, a cache group includes a corresponding cache table $T$ with the same schema, i.e., for each column $T_{\mathrm{B}} . c$ in the backend table there is a column T.c of same type (incl. unique constraints) in the cache table. (Foreign key constraints are not copied into the cache.)

\subsection{Completeness and Constraints}

For DB caching, completeness is a most important concept: Having all the records that are needed to eval- 
uate a certain predicate in the cache is known under the term predicate completeness [4]. Completeness of more complex predicates is achieved by starting with completeness of very simple equality predicates and extending them with the help of cache constraints.

Equality predicates $(\mathrm{EPs})$ of the type T.c $=v$, where $v$ is a value of column T.c, are supported by the completeness of $v$. This value $v$ is complete in a cache column $T . c$ if all records from $T_{\mathrm{B}}$ that have this value in $c$ are in the cache (in $T$ ).

A referential cache constraint $(R C C)$ is a valuebased relationship between two columns: a source column $S . a$ and a target column T.b. An RCC $S . a \rightarrow T . b$ guarantees that every value in $S . a$ (in the cache!) is complete in $T . b$. This allows an equi-join (EJ) $S . a=T . b$ to be performed in the cache, once it has been verified that the needed $S$ records (specified by other predicates such as $S . b=v$ ) are in the cache.

Basically, this procedure allows us to deal with predicates of the form $E P \wedge E J_{1} \wedge E J_{2} \wedge \cdots \wedge E J_{n}$ in the cache, where all of the equi-joins $E J$ and the equality predicate $E P_{i}$ are connected via some tables. More complex predicates that can be constructed from this simple type by con-/disjunction and by further restrictions could also be processed in the cache.

\subsection{Probing and Query Execution}

When a query reaches the cache, it has to be decided whether the query can be answered partially in the cache and what part of the query result must be fetched from the backend. Deciding on the completeness of a (partial) predicate in the cache is done in two phases:

1. For each equality predicate $T . c=v$, which compares a column T.c to a value $v$, completeness of $v$ is decided by probing the cache.

2. Starting from complete values providing entry points for the query into the cache, RCCs $S . a \rightarrow$ $T . b$ matching equality predicates of type $S . a=T . b$ in the query predicate are then used to extend the completeness to the largest predicate possible.

Probing works by issuing simple existence queries for values in some columns: You might know from prior analysis that all values in a cache column are complete (column completeness [4]), or you can leverage the RCCs by probing in their source columns. Either way, the existence of a value implies its completeness in a (possibly different) column.

Once the partial predicate that is complete in the cache has been found, it is clear that, for the tables referenced in that predicate, their cache counterparts can be used for executing the query. For the remaining tables, the original table at the backend must be accessed.

\subsection{Loading and Unloading}

Records are loaded into the cache whenever there is a hint that they will be needed in the future. Filling columns are responsible for providing these hints: As soon as specific value $v$ of a filling column $f$ is referenced in a query, $v$ is made complete in the cache and fulfilling RCCs make sure that a "neighborhood" of related records becomes available in the cache, too.

Loading is guided by the graph of RCCs: The sets of records to be inserted in the cache can be determined by following the RCCs Usually, records inserted into the source table of an RCC demand matching records to be loaded into the target table. The actual insertion of those record sets into the cache tables may be performed in the reverse order (bottom-up) to provide more consistent cache states during the loading and thus better concurrency with readers [2].

Unloading aims at reversing the process of loading but has to cope with added difficulties due to records being required via multiple RCCs.

\subsection{Prototype ACCache}

Our prototype implementation of the techniques just sketched is called ACCache (Adaptive Constraintbased Cache) [2]. It employs a middleware strategy to realize the behavior of the database cache on top of two regular databases (backend and cache) that are accessed via JDBC: Probing, (un)loading, and maintenance of RCCs are done via (prepared) SQL statements. Query processing leverages the federated-query functionality of the underlying database management system to be able to access backend as well as cache tables within a single SQL query that is a rewrite of the original user query. (To the outside, ACCache implements a JDBC interface.)

Data to be unloaded from the cache is chosen based on access statistics, but the unloading itself is not performed yet. At our current stage, we start out with an empty cache and consider only a number of loading operations and their influence on query performance.

Adaptiveness comes in two facets in ACCache: First, ACCache adapts its contents to the query workload on the instance level, i.e., only useful sets of records that will be used in the future are kept in the cache. Second, we are planning to make ACCache adaptive on the schema level: The cache group definition may be adapted, i. e., cache tables or RCCs may be added or dropped if monitoring the workload provides hints at often used join directions or at data that is often used together (either in the same query or in multiple queries that occur closely together in time). 
In the current implementation, the cache system is responsible for initiating the necessary loading actions and for deciding what data has to be loaded. This approach guarantees that the backend is not additionally burdened. Besides, for each incoming query, the probing has to be performed. In the following sections, we would like to check the behavior and the performance aspects of these two main cache functions. This allows us to reflect on our design decisions later on.

\section{Measurement Setup}

It is a well-known fact that caching dramatically improves the performance of query processing under heavy workloads and high network latency. Therefore, our main goal is not to prove this fact again, even though this is observable in our measurements, of course. We want to measure the behavior and the overhead of the main functions implemented in our cache system (probing and loading). It is not overly urgent to verify the overhead of these functions separately. On the contrary, we should look at them together and especially at the interactions between the backend and cache database under the anticipated high latency.

Measurements with a setup where there is almost no latency give us only a feeling of how much overhead the cache system generates. The more interesting part is to analyze how much time the loading process takes to enable a significant caching effect as soon as the needed data is available in the cache: Such results can help to improve the way data is loaded into the cache. In addition, the results can assist us in finding optimization rules so that we can improve the adaptivity of the cache system. For example, assuming we implement advanced loading methods, it will be possible to switch the loading method automatically to the appropriate implementation dependent on the observed latency.

\subsection{Capturing Measured Values}

For performing measurements in the ACCache system, we use a framework developed in-house [5]. It supports a developer in setting up and executing measurements for a distributed system. The framework's components offer a wide range of functionality for measuring distributed structures.

In our measurement framework we use a working node to represent an application within the distributed system we want to measure: For our first measurement, we have built three working nodes representing the backend database system, the ACCache system, and a simulated client, which generates the workload during the measurement (see also Fig. 1). To capture val- ues from an application represented by a working node, an observer needs to be defined. The captured values are associated with execution contexts that model their semantics and dependencies among each other.

For our ACCache measurements, our overall setup including the measured components (square boxes) as well as the measuring components (curved boxes), which are spread over four separate network nodes, is shown in Fig. 1. It also sketches two of our parameters that will be explained in the following: network delay and cache bypass.

\subsection{Parameters}

As it is difficult for us to actually maintain and use a backend DB in some remote part of the world, we employ a network emulator to approximate the characteristics of the network between backend and cache: NetEm is an enhancement of the traffic control facilities of the Linux kernel that allows adding delay, packet loss and other scenarios. [3]

The round-trip delay inherent in our real network between backend and cache node is about $0.2 \mathrm{~ms}$. During our measurements we raised this round-trip delay by an amount of $\mu \pm \sigma$ according to a normal distribution with a standard deviation of $\sigma=\mu / 10$ and a correlation $\rho=25 \%$. The mean round-trip delay $\mu$ was chosen from 0,40 , and $100 \mathrm{~ms}$.

\subsection{Backend Schema and Cache Group}

As a baseline, we performed all measurements a second time with our cache still in the path from client to backend but with the main caching functionality bypassed (i. e., no query analysis, probing, rewriting, etc., were performed but every query was immediately executed at the backend). In this case, our cache acted as a kind of forwarding proxy.

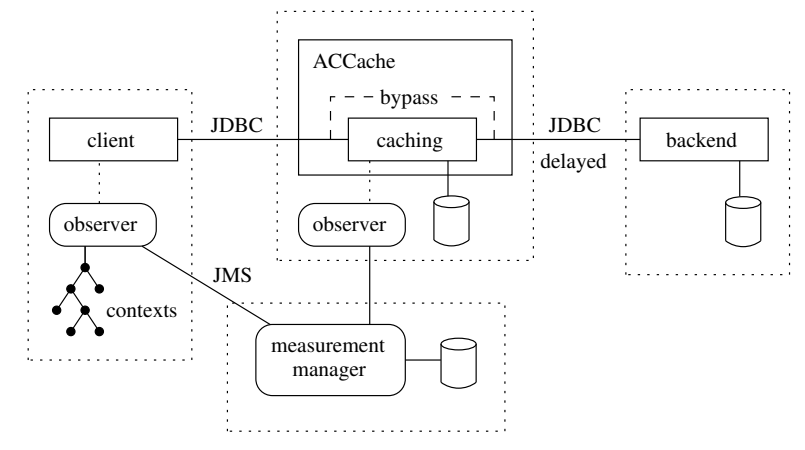

Figure 1. Measurement setup on four nodes: client, ACCache, backend, and measurement manager 
The scenario for our measurements is loosely based on the TPC-W benchmark [8], which models an online store. We use its database schema (with tables for customers, orders, items, etc.) and data in the backend DB (100000 items).

As a cache group, we use the one given in Fig. 2, which ensures that for any order loaded into the cache the corresponding order lines, addresses, and items are loaded, too. Furthermore, every item loaded into the cache will be accompanied by its author. Orders and items get into the cache only if referenced specifically by their primary keys (id columns).

\subsection{Queries: Order Display}

The queries that we pose to the cache are inspired by the web interaction "order display" of TPC-W. First of all, we display the details of a selected order including the referenced addresses:

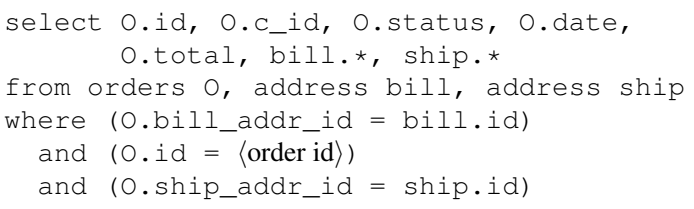

We then need a listing of all order lines belonging to that order where we include some basic information on the ordered items:

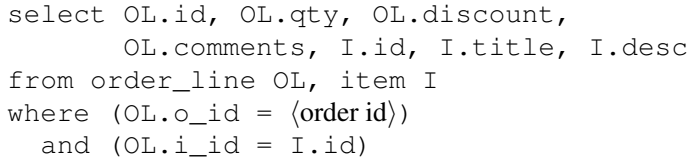

Finally, we simulate the user requesting the item details for each displayed order line in turn with multiple instances of the following statement.

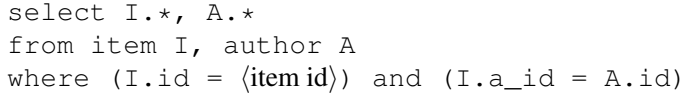

\subsection{Measured Values}

We designed two observers (for the client and cache), which transmit measured values to the manager. On the client, we have only a single execution context for executing a query. For each query, we capture three timestamps: before the query processing starts, when the first row of the query result has been fetched (firstrow time), and after fetching and printing all resulting rows (all-rows time).

For the cache, we built the execution contexts "query", "analysis", and "load". In the query context, we capture the start and end timestamp of the query

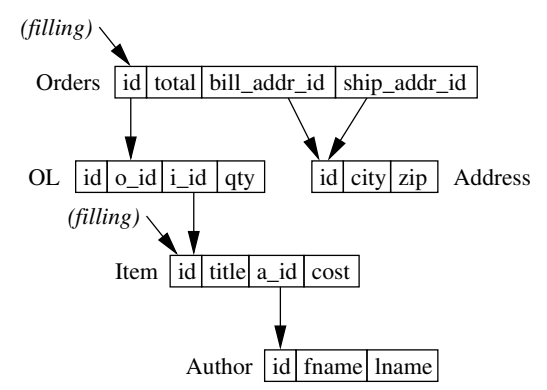

Figure 2. A cache group for the TPC-W schema [8] (with five cache tables, two filling columns $O . i d$ and I.id, and five RCCs)

processing and a reference to the client query execution context that caused the execution on the ACCache system. One of the parts of processing a query is the analysis phase (probing, query rewriting). Therefore, an analysis context is created as a child context of the query and the start and the end of this phase are captured. Furthermore, the analysis phase might decide that tuples should be loaded into the cache: Each loading job created within our system is mirrored into a load execution context. This execution context captures the start and end timestamps and, additionally, the pair of column and value that is the starting point for the loading job.

Timestamps are retrieved with Java's nanoTime method, which has an accuracy of about $\pm 3 \mu$ s on our nodes. This means that the error in calculated durations will be twice that much and thus is negligible (compared with durations of about $30 \mathrm{~ms}$ and more).

\section{Results}

In our concrete setup, we executed the work unit "order display" five times in a row per measurement run without any delays between the queries: After displaying an order $(\mathrm{O})$ and the retrieval of the corresponding order lines (OL), all of the related five items (I) were accessed. This work unit was then repeated for the very same order id.

As described above, we varied the round-trip delay between backend and cache and enabled or disabled our cache bypass: The six resulting configurations were repeated three times each, resulting in 18 measurement runs in total.

Figure 3 shows the average times spent on reading and displaying the query results in all measurement runs. The actual measured values lie within about $\pm 10 \mathrm{~ms}$ around this average. Figure 4 shows the timing and the duration of client queries and load operations at 


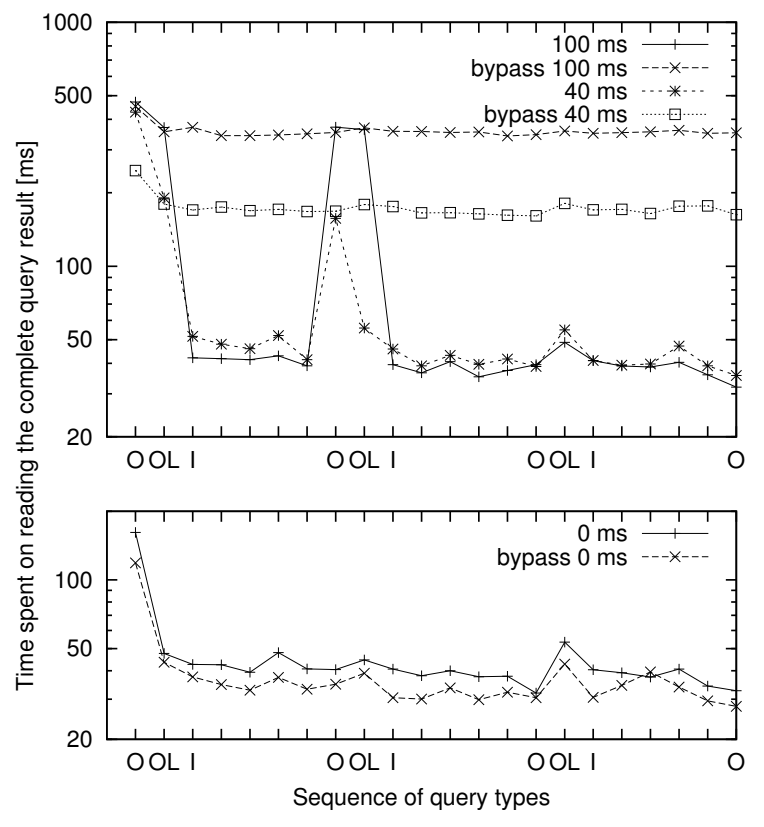

Figure 3. Query execution times as perceived by the client

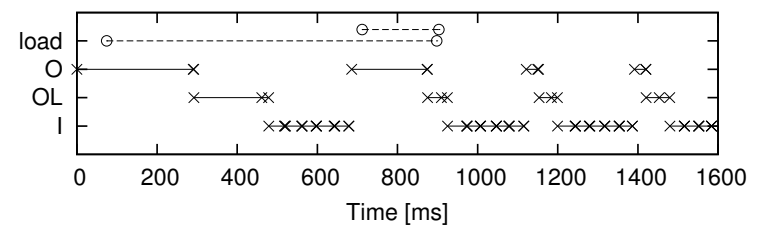

Figure 4. Query execution/loading times and sequence of events (round-trip $40 \mathrm{~ms}$, no bypass)

the cache in a selected measurement run with a roundtrip delay of $40 \mathrm{~ms}$ where the cache is not bypassed. The crosses mark significant points of time within the processing of a query, namely start of the query, the first-row time, and the all-rows time. However, these are only visible separately in the case of an order-line query (OL). The other queries deliver only one row, which makes first-row time and all-rows time almost coincide.

As expected, the cache dramatically improves the response time of the queries if the cache loading for the order under consideration has finished. Interestingly, the cache can already be used to process the first five item queries when the loading has not yet finished (compare Figs. 3 and 4). This is due to the fact that, in the current implementation, loading is performed bottom-up (as sketched in Sect. 2.3). That is, with our cache group, loading starts at the author and address tables and proceeds to the orders table. Therefore, the items related to the order requested become available

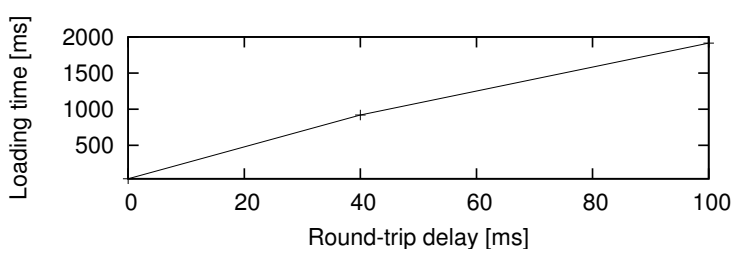

Figure 5. Cache loading times (for an order plus dependent records)

(and usable) in the cache before the order itself does. As can be seen in Fig. 4, the loading is complete shortly after the second $\mathrm{O}$ query (which corresponds to the second set of peaks in Fig. 3); from then on, all following queries benefit from the cache contents.

For reference, the lower part of Fig. 3 shows the measurement runs with no latency $(0 \mathrm{~ms})$. The difference between the runs with and without bypass clearly identifies the overhead inherent in our cache processing steps: Without delay (i. e., with a non-remote backend database), the cache needs 5 to $10 \mathrm{~ms}$ more time to answer a query than in the bypassing scenario. This is caused by the analysis phase and in particular the probing, which is always performed if a query could potentially be executed in the cache. When the delay increases, the costs involved in the probing are more than compensated by the savings due to the avoidance of remote accesses to the backend.

Figure 5 shows how the time needed to load the requested order into the cache and to fulfill the RCCs by loading dependent records changes with the (network) distance between cache and backend DB. As can be seen, the loading time increases almost linearly with the round-trip delay. With $0 \mathrm{~ms}$ delay, the cache is loaded almost instantly (after $37 \mathrm{~ms}$ ); with a delay of $100 \mathrm{~ms}$, it takes $2 \mathrm{~s}$. This might mean that in some cases the cached data could be available too late to answer queries that have occurred in the meantime.

You might wonder why there are two loading operations in Fig. 4, which actually refer to the same order. The second loading operation is initiated at a time (ca. $700 \mathrm{~ms}$ ) when the first operation has not yet succeeded in loading the order into the cache. When this first operation finishes at about $900 \mathrm{~ms}$, a quick check suffices to see that there is not any work left. (Loading operations are executed strictly sequentially at the moment.)

From this behavior, we can draw the conclusion that we should look into other ways of performing the cache loading. Moreover, we need to know or make assumptions about typical workloads and the delay between queries associated to each other via data locality; only then can we decide whether our loading is fast enough or whether loading should be coupled with 
query execution (i.e., data would simultaneously be used to answer a query and load the cache).

\section{Related Work}

Meanwhile, the most important database vendors IBM, Microsoft and Oracle have developed their own approaches to database caching in addition to existing replication methods. Recent development has shown that using cache groups is one of the standard approaches. IBM and Oracle both allow sub-table-level caching via cache groups in their prototypes/products (DBCache from IBM, Times Ten In-Memory Database from Oracle). IBM measured its prototype's functionality [1] and showed that the overhead of probing and loading has low significance: The response time increases only by up to $6 \%$ for join queries. This result compares to our observation (with a latency of $0 \mathrm{~ms}$ ). But the influence of high latency between backend and cache on the loading process is not discussed.

For the Oracle In-Memory Database TimesTen, we did not find any significant measurements. For the timing values given in the technical whitepaper [7], it is unclear to which tested functionalities they refer exactly.

Microsoft has built a caching solution called MTCache [6]. This solution does not use the concept of cache groups as its basis. Instead, materialized views are used together with standard replication methods to build a cache mechanism. Keeping subsets of base tables in the cache in a way similar to a cache group can be modeled via stacking views. Since the presented measurements of the MTCache system aim at the performance of the entire system, they cannot be directly compared with our measurements. Anyway, there is no description of the cache functions' behavior when a significant latency to the backend database exists.

\section{Conclusion}

We have subjected our ACCache prototype to a first series of measurements to get an indication of its potentials. Our results are encouraging: Already with small delays to the backend database server, our constraintbased cache is able to save query processing time, even for queries that are only related to an initiating query: Expected locality in database accesses can be conveniently modeled through cache groups, especially with cache constraints like RCCs that define an environment of related tuples.

The observed time spent on loading all needed data into the cache, which depends linearly on the latency between cache and backend, could become a problem if the latency is too high. Because we can expect a high bandwidth, another possibility to load data into the cache is, for example, to compose a single package of data related to a cache miss at the backend database: Then the backend database is responsible for resolving all RCC dependencies. But as an advantage, all of the data dependent on a cache miss can be transferred at once (perhaps bundled with the result of the initiating query) and just a notification of the cache miss may be sent to the backend.

This idea of improving the loading process, which directly results from our measurements, shows that analyzing the effects of latency cannot be neglected as earlier analyses of caching products tended to do (cf. Sect. 5). High latency is one of the fundamental assumptions in scenarios that caching is designed for; hence, there is no point in performing measurements in no-delay setups.

We also learned from these measurements that setting up a general, automated measurement environment for a distributed system is a complex and timeconsuming task. But after all, the possibility of designing well-suited execution contexts for tracing the work performed on the working nodes and their dependencies will assist us in setting up future measurement runs more quickly.

\section{References}

[1] M. Altinel, C. Bornhövd, et al. Cache tables: Paving the way for an adaptive database cache. In $V L D B, 718-729$, 2003.

[2] A. Bühmann, T. Härder, et al. A middleware-based approach to database caching. In Y. Manolopoulos, J. Pokorný, et al., eds., ADBIS 2006, vol. 4152 of LNCS, 182199. Thessaloniki, 2006.

[3] S. Hemminger. Network emulation with NetEm. In Proceedings of linux.conf.au (LCA). Canberra, 2005.

[4] T. Härder, A. Bühmann. Value complete, column complete, predicate complete - Magic words driving the design of cache groups. VLDB Journal, 2007. Online First, http://dx.doi.org/10.1007/s00778-006-0035-9.

[5] J. Klein. Development of an automated measurement environment for the constraint-based database caching (in German). Master's thesis, TU Kaiserslautern, 2006. http://wwwdvs.informatik.uni-kl.de/pubs/ DAsPAs/Kle06.DA.html.

[6] P. Larson, J. Goldstein, et al. MTCache: Transparent midtier database caching in SQL server. In ICDE, 177-189. IEEE Computer Society, 2004.

[7] Oracle. Oracle TimesTen Product and Technologies. White paper, 2007. http://www.oracle.com/technology/ products/timesten/pdf/wp/wp_timesten_t\%ech.pdf.

[8] TPC. TPC benchmark W (web commerce) specification. http://www.tpc.org/tpcw/spec/tpcw_V1.8.pdf, 2002. Version 1.8 . 